\title{
The Contact Polytope of the Leech Lattice
}

\author{
Mathieu Dutour Sikirić • Achill Schürmann • \\ Frank Vallentin
}

Received: 16 June 2009 / Revised: 16 February 2010 / Accepted: 9 May 2010 /

Published online: 3 June 2010

(C) The Author(s) 2010. This article is published with open access at Springerlink.com

\begin{abstract}
The contact polytope of a lattice is the convex hull of its shortest vectors. In this paper we classify the facets of the contact polytope of the Leech lattice up to symmetry. There are 1, 197, 362, 269, 604, 214, 277, 200 many facets in 232 orbits.
\end{abstract}

Keywords Leech lattice · Contact polytope · Conway groups · Voronoi cell

\section{Introduction}

An $n$-dimensional lattice $L$ is a discrete subgroup of the $n$-dimensional Euclidean space $\mathbb{R}^{n}$ of the form $L=\left\{\sum_{i=1}^{n} \alpha_{i} b_{i}: \alpha_{1}, \ldots, \alpha_{n} \in \mathbb{Z}\right\}$, where $b_{1}, \ldots, b_{n}$ is a basis of $\mathbb{R}^{n}$. By $\lambda(L)$ we denote the Euclidean length of nonzero shortest vectors of $L$, and we denote by $\operatorname{Min} L$ the set of shortest vectors.

Every lattice comes with two important polytopes: The contact polytope of $L$ is the convex hull of its shortest vectors

$$
\mathrm{C}(L)=\operatorname{conv}\{v: v \in \operatorname{Min} L\}
$$

M. Dutour Sikirić

Rudjer Bosković Institute, Bijenicka 54, 10000 Zagreb, Croatia

e-mail: mdsikir@irb.hr

A. Schürmann $\cdot$ F. Vallentin $(\bowtie)$

Delft Institute of Applied Mathematics, Technical University of Delft, P.O. Box 5031, 2600 GA

Delft, The Netherlands

e-mail: f.vallentin@tudelft.nl

A. Schürmann

e-mail: a.schurmann@tudelft.nl 
and the Voronoi cell of $L$ is the region of points that are closer to the origin than to other lattice points,

$$
\mathrm{V}(L)=\left\{x \in \mathbb{R}^{n}: x \cdot v \leq \frac{1}{2} v \cdot v \text { for all } v \in L\right\} .
$$

Maybe one of the most remarkable lattices is the 24-dimensional Leech lattice $\Lambda_{24}$. It has 196,560 shortest vectors which is the highest possible number in dimension 24. Its orthogonal group, i.e., the group of orthogonal transformations preserving the lattice is the Conway group $\mathrm{Co}_{0}$. It has $2^{22} \cdot 3^{9} \cdot 5^{4} \cdot 7^{2} \cdot 11 \cdot 13 \cdot 23=$ $8,315,553,613,086,720,000$ elements and is connected to many sporadic simple groups. We refer to the book [6] by Conway and Sloane for an extensive treatment of the Leech lattice.

Borcherds, Conway, Parker, Queen, Sloane [6, Chaps. 23 and 25] determine the vertices of the Voronoi cell of the Leech lattice. The Voronoi cell tiles the space $\mathbb{R}^{n}$ by translations; this gives the Voronoi cell tiling of $\mathbb{R}^{n}$. So, in the context of the Voronoi cell it is natural to consider orbits under the isometry group (the group generated by the orthogonal group of the Leech lattice together with lattice translations) acting on the Voronoi cell tiling. We denote the isometry group of the Leech lattice by $\mathrm{Co}_{\infty}$. There are 307 orbits of vertices in the Voronoi cell tiling under the action of $\mathrm{Co}_{\infty}$.

In this paper we determine the facets and their incidence relations of the contact polytope of the Leech lattice. We get the following result.

Theorem 1 There are 232 orbits of facets of $\mathrm{C}\left(\Lambda_{24}\right)$ under $\mathrm{Co}_{0}$.

The contact polytope and the Voronoi cell are related. To see this relation, we consider

$$
\mathrm{C}(L)^{*}=\left\{x \in \mathbb{R}^{n}: x \cdot v \leq \frac{1}{2} v \cdot v \text { for all } v \in \operatorname{Min} L\right\},
$$

which is the standard polar polytope scaled by a factor of $\frac{1}{2} \lambda(L)^{2}$. The faces of $\mathrm{C}(L)$ and of $\mathrm{C}(L)^{*}$ are in bijection. The bijection reverses the inclusion relation: $k$-dimensional faces of $\mathrm{C}(L)$ correspond to $(n-k)$-dimensional faces of $\mathrm{C}(L)^{*}$. In particular, vertices of $\mathrm{C}(L)^{*}$ correspond to facets of $\mathrm{C}(L)$. For these notions, we refer to the standard literature on polytope theory, e.g., the book by Ziegler [21].

We chose the scaling in the definition of $\mathrm{C}(L)^{*}$ so that it contains $\mathrm{V}(L)$. In the case of the Leech lattice some vertices of $\mathrm{V}\left(\Lambda_{24}\right)$ and $\mathrm{C}\left(\Lambda_{24}\right)^{*}$ are shared. As a side remark: One has the equality $\mathrm{C}(L)^{*}=\mathrm{V}(L)$ if and only if $L$ is a root lattice, see Rajan and Shende [18].

Theorem 2164 orbits of vertices of $\mathrm{C}\left(\Lambda_{24}\right)^{*}$ are also orbits of vertices of $\mathrm{V}\left(\Lambda_{24}\right)$. They are listed in Table 1 in the complete version of the paper [10]. The additional 68 orbits of vertices are listed in Table 2 of [10].

We classify the shared vertices in Sect. 2 and give them in Table 1 of [10]. In Sect. 3 we classify the additional vertices of $\mathrm{C}\left(\Lambda_{24}\right)^{*}$ that are not vertices of $\mathrm{V}\left(\Lambda_{24}\right)$. 
We conclude the paper by Sect. 4, where we briefly explain our computational techniques.

The data presented here is also electronically available from [8].

\section{Shared Vertices}

In this section we explain the notation used in Table 1 of [10], which contains the 164 orbits of shared vertices mentioned in Theorem 2 .

The vertices of the Voronoi cell of a lattice are centers of empty spheres, i.e., spheres $S(x,\|x\|)$ with center $x$ and radius $\|x\|$ which contain lattice points on their boundary but not in their interior. The convex hull of lattice points on the boundary of such an empty sphere is called the Delone cell of the vertex $x$.

The Delone cells of the Leech lattice are classified by Borcherds, Conway, Parker, Queen, Sloane [6, Chaps. 23 and 25] up to the action of the isometry group $\mathrm{Co}_{\infty}$. For this classification, they use Coxeter-Dynkin diagrams.

A Coxeter-Dynkin diagram with vertex-set $\{1, \ldots, N\}$ is a symmetric $N \times N$ matrix $\left(m_{i j}\right)_{1 \leq i, j \leq N}$ with ones on the diagonal and $m_{i j} \geq 2$ if $i \neq j$ and $m_{i j} \in \mathbb{N} \cup\{\infty\}$.

A Coxeter-Dynkin diagram is called simply laced if $m_{i j}=2,3$, or $\infty$. The Cartan matrix of a Coxeter-Dynkin diagram $\left(m_{i j}\right)_{1 \leq i, j \leq N}$ is the matrix $M=$ $\left(-\cos \frac{\pi}{m_{i j}}\right)_{1 \leq i, j \leq N}$. A Coxeter-Dynkin diagram is called spherical if its Cartan matrix is positive definite and affine if its Cartan matrix is positive semidefinite. A Coxeter-Dynkin diagram is called decomposable if we can partition its vertex-set into $S_{1} \cup S_{2}$ with $m_{i j}=2$ if $i \in S_{1}$ and $j \in S_{2}$. It is called indecomposable otherwise. A Coxeter-Dynkin diagram $D$ admits a unique decomposition into indecomposable Coxeter-Dynkin diagrams $D_{1}, \ldots, D_{r}$, which we write as $D=D_{1} D_{2} \ldots D_{r}$. The classification of spherical and affine Coxeter-Dynkin diagrams is presented, for example, in Humphreys [13, Sects. 2.4 and 4.7]. Here the famous $A-D-E$ diagrams show up, explained, e.g., by Hazewinkel, Hesselink, Siersma, and Veldkamp [12]. The spherical, simply laced, indecomposable Coxeter-Dynkin diagrams are $a_{n}$ for $n \geq 1, d_{n}$ for $n \geq 4$, and $e_{n}$ for $6 \leq n \leq 8$. Each diagram corresponds to an indecomposable affine diagram: $A_{n}, D_{n}$, and $E_{n}$. All these diagrams are pictured, e.g., in [6, Fig. 23.1].

In the Leech lattice, a Coxeter-Dynkin diagram $\left(m_{i j}\right)_{1 \leq i, j \leq N}$ can be associated with a Delone cell with vertex-set $\left\{v_{1}, \ldots, v_{N}\right\}$ by

$$
m_{i j}= \begin{cases}1 & \text { if }\left\|v_{i}-v_{j}\right\|^{2}=0 \\ 2 & \text { if }\left\|v_{i}-v_{j}\right\|^{2}=4, \\ 3 & \text { if }\left\|v_{i}-v_{j}\right\|^{2}=6 \\ \infty & \text { if }\left\|v_{i}-v_{j}\right\|^{2}=8\end{cases}
$$

As can been seen in Table 1 of [10], different Delone cells may have the same Coxeter-Dynkin diagram.

In Table 1 of [10] the rows are sorted first by the squared length $\|v\|^{2}$ (third column) of the vertex $v$. Second, they are sorted by the size of the stabilizer of $v$ within 
the orthogonal group of the Leech lattice (fifth column) and then by the number of incident facets of $\mathrm{C}\left(\Lambda_{24}\right)^{*}$ (fourth column).

In the second column we give the Coxeter-Dynkin diagrams of the associated Delone cell of $v$. Note that the diagrams are affine if and only if the squared length of $v$ equals 2 , the maximum among shared vertices. In all other cases they are spherical. Furthermore, in the spherical cases the number of incident facets is always equal to the minimum possible number of 24. These observations follow from [6, Chaps. 23 and 25].

In the last column we give the MOG (Miracle Octad Generator) coordinates of representatives of each orbit which one has to multiply with $\alpha$ (sixth column). The MOG coordinates form a standard coordinate system for the Leech lattice. They are explained in [6, Chap. 11].

There are 307 orbits of vertices in the Voronoi cell tiling under the action of the isometry group $\mathrm{Co}_{\infty}$ of the Leech lattice. Our computation shows that there are 5,297 orbits of vertices of the single Voronoi cell $\mathrm{V}\left(\Lambda_{24}\right)$ under the action of the smaller, finite orthogonal group of the Leech lattice; 164 of them are shared with $\mathrm{C}\left(\Lambda_{24}\right)^{*}$.

\section{Additional Vertices}

There are 68 additional orbits of vertices of $\mathrm{C}\left(\Lambda_{24}\right)^{*}$ that are not vertices of the Voronoi cell of the Leech lattice. These additional vertices are characterized by the fact that the distance to a closest lattice point is strictly less than the distance $\|v\|$ to the origin.

Table 2 of [10] describes these 68 orbits. Like in Table 1 of [10], the rows are sorted (in this order) by the squared length $\|v\|^{2}$ (third column), the size of the stabilizer of $v$ within the orthogonal group of the Leech lattice (fifth column), and then by the number of incident facets (fourth column).

In the second column we give names for diagrams. The first row corresponds to an exceptional vertex which we explain below. The other 67 rows correspond to graphs which we define later in Sect. 3.2.

\subsection{The Exceptional Vertex}

The first orbit of vertices is exceptional: Its squared norm $8 / 3=2.666 \ldots$ is substantially bigger than the squared norm of all other vertices which lie in the interval $[1.92,2.25]$. Its incidence number of 552 and the size of its stabilizer, which is the Conway group $\mathrm{Co}_{3}$, are also substantially larger than the values for the other vertices. This orbit of vertices is a scaled copy of the vectors of $\Lambda_{24}$, having Euclidean norm $\sqrt{6}$.

In the contact polytope $\mathrm{C}\left(\Lambda_{24}\right)$ this exceptional vertex corresponds to a facet. Since it has maximum norm among all vertices, the corresponding facet is closest to the origin and has the largest possible circumsphere among all other facets of $\mathrm{C}\left(\Lambda_{24}\right)$. This solves a conjecture of Ballinger, Blekherman, Cohn, Giansiracusa, Kelly, and Schürmann [2, Sect. 3.7]. We note that a similar calculation as the one presented here, solves the corresponding conjecture about the contact polytope of the 23-dimensional lattice $O_{23}$, the shorter Leech lattice, which has 4,600 vertices. 
The 23-dimensional point configuration, given by the 552 shortest vectors of the Leech lattice defining facets incident to the exceptional vertex, appears in several different contexts: It is universally optimal (Cohn and Kumar [5]), it defines 276 equiangular lines (Lemmens and Seidel [15]), and it defines an extreme Delone cell (Deza and Laurent [14, Chap. 16.3]). Moreover, it contains a wealth of remarkable substructures (see Cohn et al. [2]), e.g., the highly-symmetric point configurations discussed in the next section, and also others, e.g., the one defined by the McLaughlin graph.

\subsection{The Other Vertices}

To the remaining 67 orbits of vertices we associate a diagram as follows. Let $v$ be one of these vertices, and let $w_{1}, \ldots, w_{N}$ be shortest vectors of the Leech lattice defining facets incident to $v$. Only the two inner products 1 and 2 occur between distinct vectors $w_{i}$ and $w_{j}$. So we can define a graph with vertex-set $\{1, \ldots, N\}$ and edge-set $\left\{\{i, j\}: w_{i} \cdot w_{j}=1\right\}$; the other inner product 2 defines nonedges.

Here again the graphs decompose into connected components where several of these occurring components are highly symmetric and have been studied in other contexts. We discuss them below; the graphs $a_{n}, d_{n}$, and $e_{n}$ are already described in the previous section, and the remaining ones are in Fig. 1.

The Higman-Sims graph $\mathrm{HS}_{100}$ is the unique strongly regular graph with parameters $(100,22,0,6)$. See Brouwer, Cohen, and Neumaier [4, Chap. 13.1].

The Hoffman-Singleton graph $\mathrm{HS}_{50}$ is the unique strongly regular graph with parameters $(50,7,0,1)$. See [4, Chap. 13.1].

For the Johnson graph $J(7,4)$, see [4, Chap. 9.1].

A $(k, g)$-cage is a regular graph of valency $k$ and girth $g$ which attains the minimum possible number of vertices. The $(5,6)$-cage (incidence graph of a projective plane $\mathrm{PG}(2,4))$ and the $(3,8)$-cage (Tutte-Coxeter graph) are unique. See [4, Chap. 6.9] and Tutte [20].

The Coxeter graph Cox is the unique distance regular graph with intersection array $\{3,2,2,1 ; 1,1,1,2\}$. See [4, Chap. 12.3].

In Fig. 1 we list the remaining graphs. The vertices of these graphs only have degree one (white circles), degree two (sitting on edges, which are not depicted; see below), or degree three (black circles). We have three kinds of trees: $T_{b}^{a} c$ having $a+b+c+4$ vertices, $T_{b}^{a} c_{e}^{d}$ having $a+b+c+d+e+6$ vertices, and $T_{b}^{a} c^{d} e_{g}^{f}$ having $a+b+c+d+e+f+g+8$ vertices; we have 12 other graphs $G_{n, m}$ with $n$ vertices and $m$ edges. In Fig. 1 the numbers on the edges show how many vertices of degree 2 sit on them, but in the following four cases we did not put these numbers: The graph $G_{24,30}$ has one vertex of degree 2 on every edge, $G_{25,30}$ is the Petersen graph which has one vertex of degree 2 on every edge, $G_{22,22}$ has three vertices of degree 2 on every edge, and the graph $G_{24,27}$ is the complete bipartite graph $K_{3,3}$ which has two vertices of degree 2 on every edge.

\section{Computational Techniques}

Computing the vertices of $\mathrm{C}\left(\Lambda_{24}\right)^{*}$ from its facets is called a polyhedral representation conversion problem. A direct application of standard programs like Fukuda's 


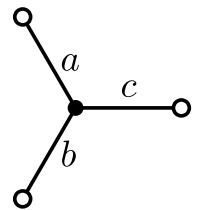

$T_{b}^{a} c$

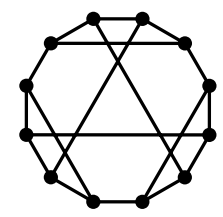

$G_{24,30}$

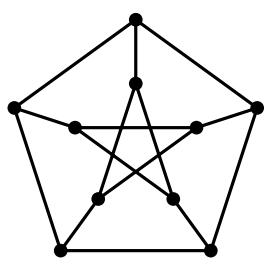

$G_{25,30}$

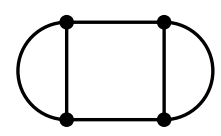

$G_{22,22}$

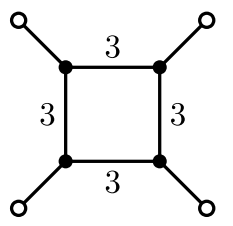

$G_{20,20}$

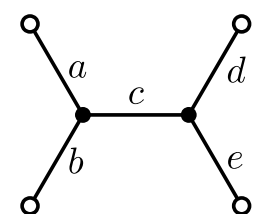

$T_{b}^{a} c_{e}^{d}$

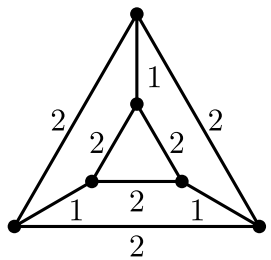

$G_{21,24}$

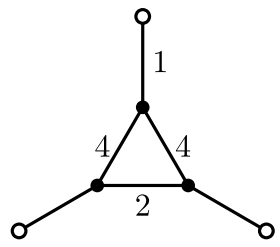

$G_{17,17}$

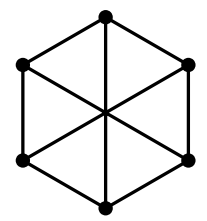

$G_{24,27}$

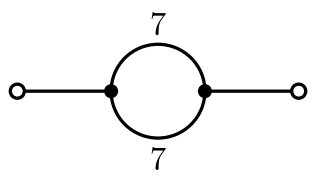

$G_{18,18}$
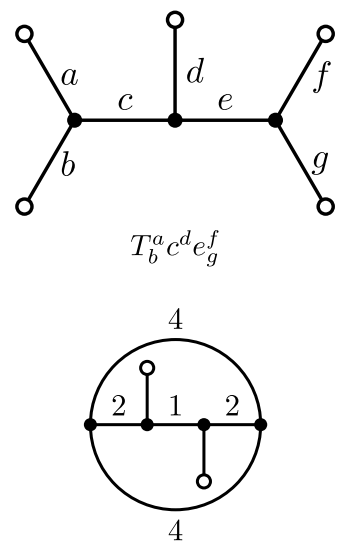

$G_{19,20}$

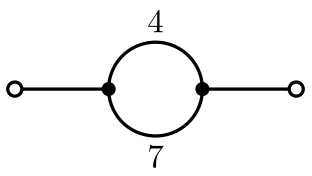

$G_{15,15}$

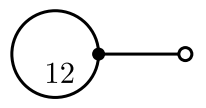

$G_{14,14}$

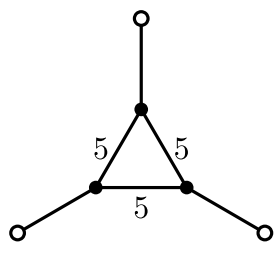

$G_{21,21}$

Fig. 1 Diagrams

cdd [11] or Avis' lrs [1] for this conversion is not feasible due to the extremely large number of vertices.

In order to exploit the symmetries of $\mathrm{C}\left(\Lambda_{24}\right)^{*}$, we use the adjacency decomposition method which is surveyed in Bremner, Dutour Sikirić, and Schürmann [3]. An implementation by the first author is available from [7]. 
The adjacency decomposition method computes a complete list of inequivalent vertex representatives. First, one computes an initial vertex by solving a linear program and inserts it into the list of orbit representatives. From any such representative, we compute the list of adjacent vertices, and if they give a new orbit, we insert it into the list of representatives. After finitely many steps all orbits have been treated. Computing adjacent vertices is a lower-dimensional representation conversion problem. So this method can be applied recursively.

For $\mathrm{C}\left(\Lambda_{24}\right)^{*}$, we had to come up with two case-specific insights:

From [2] it is known that the exceptional vertex of Sect. 3.1 is indeed a vertex of $\mathrm{C}\left(\Lambda_{24}\right)^{*}$. We used it as starting vertex of the adjacency decomposition method.

For checking isomorphy and for computing stabilizers, we used the following standard strategy: we characterize a vertex of $\mathrm{C}\left(\Lambda_{24}\right)^{*}$ by the set of its incident facets, and we represent the symmetry group $\mathrm{Co}_{0}$ as a permutation group acting on the 196,560 shortest vectors of the Leech lattice. Then, we use the backtracking algorithm by Leon $[16,17]$ implemented in [19]. This worked reasonably fast for all the cases except for the two orbits of vertices having the same Coxeter-Dynkin diagram $a_{1}^{25}$. The stabilizer of the corresponding Delone cell under the isometry group $\mathrm{Co}_{\infty}$ is the Mathieu group $M_{24}$. Under the action of $M_{24}$, the 25 vertices of the Delone cell split into two orbits of size 1 and 24 . Hence, these two orbits correspond to two distinct orbits of vertices of $\mathrm{C}\left(\Lambda_{24}\right)^{*}$, one having stabilizer $M_{24}$ and the other having stabilizer $M_{23}$. The backtracking algorithm of GAP could not decide in reasonable time whether or not two vertices with the same Coxeter-Dynkin diagram $a_{1}^{25}$ are in the same orbit. So we used the third method of Sect. 3.5 of [9] to resolve this problem.

Acknowledgements We started this research during the Junior Trimester Program (February 2008April 2008) on "Computational Mathematics" at the Hausdorff Institute of Mathematics (HIM) in Bonn. Then, part of this research was done at the Mathematisches Forschungsinstitut Oberwolfach during a stay within the Research in Pairs Programme from 3 May 2009 to 16 May 2009. We thank both institutes for their hospitality and support. The work of the first author has been supported by the Croatian Ministry of Science, Education and Sport under contract 098-0982705-2707. The second and the third authors were supported by the Deutsche Forschungsgemeinschaft (DFG) under grant SCHU 1503/4-2.

Open Access This article is distributed under the terms of the Creative Commons Attribution Noncommercial License which permits any noncommercial use, distribution, and reproduction in any medium, provided the original author(s) and source are credited.

\section{References}

1. Avis, D.: A C-implementation of the reverse search vertex enumeration algorithm. School of Computer Science, McGill University, Montreal, Canada 1993. http://www-cgrl.cs.mcgill.ca/ avis/C/lrs. html (Software)

2. Ballinger, B., Blekherman, G., Cohn, H., Giansiracusa, N., Kelly, E., Schürmann, A.: Experimental study of energy-minimizing point configurations on spheres. Exp. Math. 18, 257-283 (2009)

3. Bremner, D., Dutour Sikirić, M., Schürmann, A.: Polyhedral representation conversion up to symmetries. In: Proceedings of the 2006 CRM Workshop on Polyhedral Computation. AMS/CRM Lecture Notes, vol. 48, pp. 45-71 (2009)

4. Brouwer, A.E., Cohen, A.M., Neumaier, A.: Distance-Regular Graphs. Springer, Berlin (1989)

5. Cohn, H., Kumar, A.: Universally optimal distribution of points on spheres. J. Am. Math. Soc. 20, 99-148 (2007)

6. Conway, J.H., Sloane, N.J.A.: Sphere Packings, Lattices and Groups, 3rd edn. Springer, Berlin (1999) 
7. Dutour Sikirić, M.: Polyhedral package. http://www.liga.ens.fr/ dutour (Software)

8. Dutour Sikirić, M.: Contact polytopes of lattices. http://www.liga.ens.fr/ dutour

9. Dutour Sikirić, M., Schürmann, A., Vallentin, F.: Complexity and algorithms for computing Voronoi cells of lattices. Math. Comput. 267, 1713-1731 (2009)

10. Dutour Sikirić, M., Schürmann, A., Vallentin, F.: The contact polytope of the Leech lattice (complete version). arXiv:0906.1427 [math.MG]

11. Fukuda, K.: cdd+ reference manual. Institute for operations research, Swiss Federal Institute of Technology, Zürich, Switzerland, 1995. http://www.ifor.math.ethz.ch/ fukuda/cdd_home/cdd.html (Software)

12. Hazewinkel, M., Hesselink, W., Siersma, D., Veldkamp, F.D.: The ubiquity of Coxeter-Dynkin diagrams (an introduction to the $A-D-E$ problem). Nieuw. Arch. Wisk. 25, 257-307 (1977)

13. Humphreys, J.E.: Reflection Groups and Coxeter Groups. Cambridge University Press, Cambridge (1990)

14. Laurent, M., Deza, M.: Geometry of Cuts and Metrics. Springer, Berlin (1997)

15. Lemmens, P.W., Seidel, J.J.: Equiangular lines. J. Algebra 24, 494-512 (1973)

16. Leon, J.S.: Permutation group algorithms based on partitions, I: Theory and algorithms. J. Symb. Comput. 12, 533-583 (1991)

17. Leon, J.S.: Partitions, refinements, and permutation group computation. In: Groups and Computation II. DIMACS Ser. Discrete Math. Theoret. Comput. Sci., vol. 28, pp. 123-158 (1997)

18. Rajan, D.S., Shende, A.M.: A characterization of root lattices. Discrete Math. 161, 309-314 (1996)

19. The GAP Group: GAP_Groups, Algorithms, and Programming, Version 4.4.6, 2005. http://www. gap-system.org (Software)

20. Tutte, W.T.: A family of cubical graphs. Proc. Camb. Philos. Soc. 43, 459-474 (1947)

21. Ziegler, G.M.: Lectures on Polytopes. Springer, Berlin (1995). 